\title{
Espacio y educación: desafíos de la enseñanza a distancia en el contexto de la pandemia por COVID 19*
}

\author{
Space and education: challenges of distance learning in the \\ context of the COVID 19 pandemic
}

\author{
Espaço e educação: desafios da educação a distância no \\ contexto da pandemia COVID 19
}

Recebido em 07-05-2021

Modificado em 26-07-2021

Aceito para publicação em 20-08-2021

\section{https://doi.org/10.47456/simbitica.v8i3.36815}

\section{Julio Roberto Labraña Vargas}

Ph.D. en Sociología de la Universidad Witten/Herdecke, Alemania. Licenciado en Sociología de la Universidad de Concepción y Magíster en Análisis Sistémico aplicado a la Sociedad de la Universidad de Chile, Chile. Académico del Departamento de Educación, Facultad de Educación y Humanidades de la Universidad de Tarapacá, Chile. E-mail: juliolabranavargas@gmail.com

\section{María Antonieta Urquieta Álvarez}

Ph.D. en Trabajo Social y Políticas Comparadas de Bienestar Social de la UANL, México. Licenciada en Trabajo Social de la Universidad de Valparaíso y Magíster en Trabajo Social de la Universidad de Concepción, Chile. Académica del Departamento de Trabajo Social de la Universidad de Chile y Coordinadora del Núcleo I+D Sistemas Territoriales Complejos. E-mail: antonieta.urquieta@uchile.cl

\section{(D) Sofia Adela Salinas Fuentealba}

Licenciada en Antropología Social y Magíster en Análisis Sistémico aplicado a la Sociedad de la Universidad de Chile, Chile. Investigadora del Núcleo I+D Sistemas Territoriales Complejos. E-mail: sofia.salinas@uchile.cl

\footnotetext{
Texto elaborado en el marco y con el apoyo del Proyecto 11200429 "Las tensiones en el gobierno de las universidades estatales en el sistema chileno de educación superior entre 1990 y 2020: un enfoque de cambio organizacional" y el Fondo de Incentivo a la Investigación en Docencia de Pregrado FIDOP - Proyecto FIDOP 2019-4_FACSO: "Trayectorias espaciales y sus implicancias académicas en estudiantes de primer ciclo de la Facultad de Ciencias Sociales: desafíos de equidad para la formación de pregrado".
} 


\section{Resumen}

La pandemia por COVID 19 ha alterado notablemente el funcionamiento de las instituciones educativas. La mayoría de los gobiernos han adoptado estrategias de distanciamiento social y de cuarentena, siendo la suspensión de clases presenciales una de las medidas más frecuentemente empleadas. El presente ensayo busca abordar la educación en línea empleando elementos de la teoría de sistemas sociales desarrollada por Niklas Luhmann. Según nuestro análisis, dichas modalidades de enseñanza enfrentan al menos dos retos: a) la ausencia de un espacio de interacción específicamente educativo y diferente del espacio familiar y b) la restricción de la enseñanza a aquello que puede ser transmitido digitalmente. Lo anterior demanda la necesidad de promover procesos organizacionales en las instituciones educativas que reconozcan las posibilidades de la enseñanza a distancia.

Palabras clave: educación en línea; interacción; procesos formativos; teoría de sistemas sociales.

\section{Introducción}

La pandemia por COVID 19 ha alterado notablemente el funcionamiento de las instituciones educativas. La mayoría de los gobiernos alrededor del globo han adoptado diversas estrategias de distanciamiento social y de cuarentena, siendo la suspensión de clases presenciales una de las medidas más frecuentemente empleadas. Como resultado, estudiantes y docentes de diferentes niveles educativos han debido durante los últimos meses enfrentarse a una acelerada transición a modalidades de enseñanza a distancia de emergencia, fundadas principalmente en metodologías de aprendizaje en línea (Aguilera Hermida, 2020; Reimers; Schleicher, 2020; Carrillo; Flores, 2020; Quintana Avello, 2020).

No sorprende que, desde la literatura especializada, la pandemia por COVID 19 haya ido de la mano de un renovado interés sobre las posibilidades de la enseñanza en línea. Si se examinan los estudios sobre este tema es posible identificar dos líneas de análisis claramente diferenciadas. Por una parte, investigaciones sobre la dimensión técnica de estas transformaciones, así como los desafíos que enfrenta su implementación en términos de alfabetización digital, infraestructura tecnológica y adaptación del currículo por parte de estudiantes, docentes y directivos, y distintos sectores de la población (Horvitz, 2017; Adedoyin; Soykan, 2020). Por otra, estudios que, posicionándose normativamente en el debate entre educación presencial versus educación en línea, subrayan, desde una perspectiva favorable, su potencial de democratización del conocimiento y, desde enfoques más críticos, su carácter inherentemente insuficiente frente a modalidades de enseñanza cara-a-cara (Larreamendy-Joerns; Leinhardt, 2006; Giroux, 2014; Shah, 2016). 
El presente ensayo busca abordar la educación en línea empleando elementos de la teoría de sistemas sociales desarrollada por Niklas Luhmann. Si se sigue la hipótesis desarrollada por este autor, existen razones estructurales que avalan la primacía de la interacción cara-a-cara en los procesos formativos derivados, como exploraremos aquí, de la diferenciación de un sistema centrado en provocar cambios en los seres humanos.

Para desarrollar esta argumentación, así como explorar sus rendimientos para la comprensión del vínculo entre educación e interacción cara-a-cara, este estudio se organiza en las siguientes secciones. En primer lugar, introducimos la caracterización del sistema de la educación desde la teoría de sistemas sociales, explorando cómo su deriva evolutiva reciente en la sociedad moderna ha ido asociada, en ausencia de mecanismos probabilizadores alternativos, en la dependencia del éxito de sus comunicaciones de la interacción mediada organizacionalmente. A continuación, una vez identificadas las principales características de este sistema, empleamos este enfoque para explorar los atributos diferenciadores de la enseñanza a distancia. Según nuestro análisis, dichas modalidades de enseñanza - considerando las herramientas de docencia a distancia usadas generalmente hoy en día - enfrentan al menos dos retos: a) la ausencia de un espacio de interacción específicamente educativo y diferente del espacio familiar y b) la restricción de la enseñanza a aquello que puede ser transmitido digitalmente (hoy principalmente aquello que puede ser inmediatamente visto u oído). En tercer lugar, discutimos cómo estos retos, especialmente en el contexto de la actual pandemia, plantean la necesidad de promover procesos organizacionales de reflexión, a nivel de todo tipo de instituciones educativas, que no aspiren simplemente a replicar las características de la enseñanza cara-a-cara en un entorno digital, sino que reconozcan sus posibilidades y desafíos. El artículo finaliza con un resumen y líneas para futuros estudios.

\section{Educación e interacción desde la teoría de sistemas sociales}

La teoría de sistemas sociales, en su versión desarrollada por Niklas Luhmann, considera que la sociedad moderna se caracteriza por la primacía de una diferenciación funcional. En este sentido, a diferencia de sociedades anteriores, con formas predominantes de diferenciación por segmentos o estratos, la sociedad moderna se caracteriza por la emergencia de sistemas parciales, cada uno de ellos especializado en el cumplimiento de una función, y que operan de modo autónomo frente a los demás (Luhmann, 2007).

La educación es uno de estos sistemas. A diferencia de la formación en sociedades tradicionales, en que las labores pedagógicas son responsabilidad de las propias familias y 
abarcan conocimientos relacionados con sus futuras posiciones, en el caso de las clases altas, y con valores eclesiásticos, en el caso de las clases bajas, en la sociedad moderna la educación se extiende en principio de manera igualitaria al conjunto de la población (Luhmann, 1993; Vanderstraten, 2004a; Tyrell; Vanderstraeten, 2007).

Si bien dicho proceso ocurre a diferentes velocidades en distintas regiones del globo, la emergencia y expansión de las instituciones educacionales es un proceso que adquiere una dinámica propia, en especial entre los siglos XVIII y XIX. Dicha aceleración va de la mano de tres fenómenos que consolidan el proceso de diferenciación de este sistema parcial. Primero, la emergencia del concepto del niño ${ }^{1}$, ya no comprendido como un adulto en miniatura, sino como un individuo cuyo desarrollo debe ser impulsado a través de métodos adecuados que atiendan a sus características distintivas. A continuación, la profesionalización de la docencia, fenómeno asociado a la desvalorización de la capacidad de enseñanza de las familias, por una parte, y la creciente demanda por especialistas que, conocedores de los atributos particulares de los niños, fuesen capaces de asegurar que efectivamente estos aprendiesen. Finalmente, el establecimiento de la educación primaria obligatoria y la subsecuente construcción de una red inicial de escuelas, apoyada usualmente por el Estado en oposición del poder de los sectores industriales y las congregaciones religiosas, lo cual hizo posible extender la formación a sectores antes excluidos, separando físicamente a los estudiantes de sus familias y ubicándoles en espacios diferenciados estructural y funcionalmente con el fin de formarlos en ideas comunes (Luhmann, 1993; Luhmann; Schorr, 2000; Luhmann, 2002).

De esta manera se constituye el sistema educativo moderno: con niños en necesidad de ser educados por docentes poseedores de las herramientas pedagógicas adecuadas en espacios físicos diferentes de aquellos donde usualmente residen con sus familias. Si se examina históricamente, esta estructura de base se ha mantenido en sus fundamentos en los últimos siglos. Aunque ciertamente la reflexión educativa ha evolucionado acorde a los tiempos - ya no se suele considerar la violencia como una herramienta pedagógica adecuada, por ejemplo -, la relación a nivel de la sala de clases apenas ha cambiado. La estructura básica de dicha relación, esto es, la organización de la interacción en un espacio físico en que las oportunidades de habla están distribuidas asimétricamente y orientadas al aprendizaje y evaluación de los alumnos se mantienen aún como la forma usual de educar con más o menos variaciones en términos de flexibilidad en la aplicación de las reglas y amplitud de las materias consideradas como relevantes (Luhmann, 2002).

\footnotetext{
${ }^{1}$ Usamos el concepto masculino genérico de niño dado que la distinción semántica generalizada de niño y niña es muy posterior a los procesos de diferenciación descritos en este punto.
} 
La constancia de dicha estructura de interacción situada organizacionalmente no es casualidad según Luhmann. De acuerdo con el sociólogo alemán, la educación, a diferencia de otros procesos de socialización, se estructura como una socialización intencionada que no puede restringirse al mero uso de las causalidades que puedan ocurrir en el transcurso de la interacción orientado al aprendizaje de modos de comportamiento en situaciones restringidas (Luhmann, 1998). Si bien dicha opción podía ser suficiente en sociedades con menor movilidad social, en el contexto de la sociedad moderna la educación responde al problema de cómo generar premisas para contactos que de otra manera serían improbables y asegurar la existencia - al menos en un nivel operativo - de un consenso para el comportamiento en diferentes contextos sociales ${ }^{2}$.

El proceso educativo así descrito se constituye en una oferta que este sistema parcial produce y que solo es seleccionada por sus destinatarios si es que estos la consideran relevante. Desde este punto de vista, el cumplimiento de la función formativa es por definición improbable: precisamente por su carácter intencionado, la educación genera automáticamente resistencias entre individuos que pueden simplemente optar por no prestarle atención. Basta pensar acá en las posibilidades de éxito tras detener a un niño en la calle e intentar enseñarle las tablas de multiplicar. En este sentido, a diferencia de otros esfuerzos de transformación de personas, como una relación de pareja o una terapia, en el caso de la educación no es posible presuponer la motivación de los participantes para participar en dicho esfuerzo. Mientras en estos casos la motivación puede ser asumida por las relaciones de afecto o el interés en recibir ayuda profesional para superar una crisis biográfica, los estudiantes suelen encontrarse en una situación en que son forzados a aprender nuevas materias en las que no tienen interés evidente, lo explica la evidente dificultad propia de las tareas pedagógicas (Vanderstraeten, 2004b).

El manejo de esta improbabilidad permite comprender la predilección característica de este sistema por la interacción situada organizacionalmente. Las interacciones son sistemas sociales cuyos límites de sentido son los de la presencia: como tales se basan en la percepción recíproca de sus participantes que asegura una simultaneidad basal de irritaciones para los distintos sistemas psíquicos participantes (Luhmann, 1975). Si bien dichas observaciones son interpretadas de manera diferenciada al nivel de estos últimos sistemas, crean a la vez oportunidades para una intensificación del proceso comunicativo que puede aumentar las posibilidades de éxito de la educación, esto es, el cumplimiento de sus esfuerzos de cambio de los seres humanos. En tal sentido, debido a la existencia de una continua atención en la interacción no es posible no comunicar en este tipo de sistemas, de lo que resulta una expansión de oportunidades de transformación de sistemas psíquicos (Luhmann, 2004a).

\footnotetext{
${ }^{2}$ Para profundizar sobre este punto veáse especialmente los trabajos de Vanderstraeten $(2002 ; 2020)$.
} 
La relevancia de la interacción situada se explica entonces porque probabiliza la función formativa del sistema educativo, condición que la suspensión y/o reemplazo de las actividades presenciales, característico de la actual docencia en el contexto de la pandemia, suprime total o parcialmente y abre interrogantes que a continuación pasamos a discutir.

\section{La enseñanza en línea y sus diferencias respecto de la formación cara-a-cara}

Si se considera lo anterior, es posible ahora examinar con mayor detalle las diferencias entre la interacción pedagógica cara-a-cara y las posibilidades de la enseñanza en línea. En particular, argumentaremos que esta enfrenta dos restricciones: la ausencia de ruptura entre espacio familiar y educativo y la restricción de la enseñanza a aquello transmisible digitalmente con el estrechamiento de las oportunidades de autoformación.

\section{La espacialidad del aprendizaje cara-a-cara y los límites de la digitalización}

Como ya mencionamos, una de las principales innovaciones en la transición desde sociedades tradicionales a modernas es el traspaso de las funciones educativas desde las familias a las escuelas. Dicha transformación fue asociada a un fuerte debate normativo sobre la conveniencia de dejar el cuidado de los niños a individuos que carecen de lazos de sangre con ellos el cual fue resuelto, en la mayoría de los países, por la acción directa de los Estados y la consecuente implementación de la educación primaria obligatoria.

Dicha decisión fue asociada a la apertura de nuevas instituciones de educación financiadas principalmente por el Estado. Estas organizaciones operaban de manera autónoma, identificando los contenidos que los niños debían aprender y los métodos adecuados para su evaluación, agrupando los estudiantes generalmente según su edad y nivel de conocimientos y sometiéndolos a una temporalidad diferente de la familiar, con periodos de trabajo y recreo predefinidos e independientes de sus intenciones específicas.

Uno de los principales atributos de estas organizaciones es la ruptura que posibilitan respecto de los espacios familiares. En efecto, como ya notase Parsons (1959), la inclusión de los estudiantes en las escuelas conlleva su sujeción a dinámicas diferentes a las del espacio familiar. En particular, implica su consideración no ya como un integrante de la familia sino como un estudiante entre otros que debe aprender una serie de contenidos y cuyo grado de conocimiento debe en adelante ser evaluado en adelante según criterios uniformes. 
A su vez, la incorporación del estudiante en el espacio educativo implica un aspecto quizá más importante que es la creación de posibilidades de interacción con pares que no están aseguradas por la mera perteneciente a una familia (Vanderstraeten, 2002; 2004c). En efecto, el estudiante se ve forzado en este contexto a establecer contactos con individuos que no conoce previamente y de maneras sobre las cuales sus padres no tienen una capacidad de control directa. Como resultado de lo anterior, la escuela posibilita la creación de culturas estudiantiles diferentes a las oficiales de la organización, ayudando a consolidar en el proceso la identidad de los estudiantes y adquirir competencias afectivas de un modo que no sería posible de permanecer estos exclusivamente en sus hogares.

En ausencia de esta ruptura, como es el caso de las modalidades de enseñanza en línea, el espacio educativo no se diferencia marcadamente del espacio familiar. Si bien la evidencia disponible sugiere que se han establecido diferentes medidas en los hogares para asegurar la existencia de esta distinción en el contexto de la pandemia por COVID 19, principalmente mediante la creación de rutinas y la identificación de espacios para el trabajo en línea, esto no ha dejado de alterar los procesos de enseñanza y aprendizaje debido a la limitación de las posibilidades de intensificar la comunicación educativa y probabilizar sus fines (Bhagat; Kim, 2020; Heyang; Martin, 2020; Winters; Patel, 2021).

En efecto, por una parte, la ausencia de una ruptura respecto del espacio familiar implica mayores posibilidades de distracción para los estudiantes en sus hogares. Esto contrasta con la monotonía de la educación cara-a-cara donde, gracias al carácter usualmente cerrado de las salas de clases respecto de sus entornos físicos inmediatos, es posible aislar al docente y sus alumnos respecto de otro tipo de irritaciones y asegurar un marco mínimo de concentración ayudado por la regulación continua del docente del aprendizaje.

Por cierto, la ausencia de ruptura respecto del espacio familiar tiene diversas implicancias dependiendo del nivel educativo en que ocurre. En los niveles iniciales el "conectarse" a las clases desde los espacios familiares cotidianos suele ir asociado a una capacidad de concentración reducida como resultado de la presencia de múltiples distractores (juguetes, mascotas, personas, etc.), en tanto en el caso de los niveles secundarios y terciarios se comienzan a presenciar tensiones ya no solo en el plano de la concentración, sino también en torno a la imposibilidad de diferenciar las labores educativas con otras tareas y responsabilidades al interior del hogar como, por ejemplo, las de cuidado. Las diferencias de género no hacen sino agudizar los problemas que la formación enfrenta en este sentido.

Por otra parte, la percepción de ser percibido, atributo central de la interacción cara-acara, decae en su intensidad, especialmente en el lado de los estudiantes. La pantalla restringe 
para ellos la relevancia inmediata de los demás, pues ahora, de una manera que no es fácilmente alcanzable en el marco de la interacción tradicional, se les hace posible dedicar fácilmente su atención a otras tareas por la ausencia de una ruptura explícita con las irritaciones del hogar. Si bien dicha alternativa es en principio realizable también en el lado del docente, precisamente la presión de educar y evaluar si lo enseñado fue aprendido hace más difícil de ese lado la realización de otras actividades.

En un sentido similar con lo anterior, los estudiantes tienen a su vez menores posibilidades de interacción entre sí. Mientras en contextos cara-a-cara las interacciones entre estudiantes están más o menos aseguradas por su copresencialidad en un espacio físico común, tanto en periodos de clases como en los intervalos de recreo, en contextos de aprendizaje en línea dichos intercambios están generalmente restringidos a comunicaciones paralelas en redes sociales, perdiéndose toda la riqueza de la información no textual inmediata y su relevancia en los procesos de construcción de identidad. Dicha pérdida parece ser especialmente grave dentro de los procesos formativos de nivel inicial y, en niveles educativos superiores, en la formación profesional con foco en áreas prácticas (Coolican; Borras; Strong, 2020; Zakharova; Vilkova; Egorov, 2021; Atiles, 2021; Formosinho, 2021).

\section{La restricción de la enseñanza a lo transmisible digitalmente}

En una dirección similar, es necesario destacar que una de las claves de la presencialidad de la enseñanza es el flujo continuo de información para la percepción - entre profesores y estudiantes y entre estudiantes entre sí - que ésta posibilita. En este sentido, la interacción caraa-cara permite percibir, además del contenido explícito de la comunicación, el movimiento del cuerpo de los involucrados, con todas las señales físicas, voluntarias e involuntarias, asociadas, la comunicación proxémica (Kožić; Globočnik; Bakić-Tomić, 2013; Pietraß, 2017). A su vez, dicha forma de interacción genera también información auditiva - no solo entonaciones de voz, sino también murmuraciones y señales de asentimiento apenas registrables en el formato digital actual - que es empleada en la práctica como un indicador de la comprensión o no comprensión de los contenidos de enseñanza. Finalmente, la interacción cara-a-cara otorga la oportunidad de usar otros recursos de la percepción, como el tacto, gusto u olfato, centrales especialmente para asegurar el éxito de la formación inicial y en áreas aplicadas de la educación técnica de nivel superior que dependen especialmente de estas sensaciones.

Además de este flujo de información, la interacción cara-a-cara va inevitablemente asociada a la recursividad de la percepción visual, esto es, la imposibilidad de ver sin 
simultáneamente exponerse a la vista de los demás. Para el profesor esto resulta en el reconocimiento de que su actuación es continuamente evaluada por los estudiantes y el resultante desafío de tener que determinar si acaso su intención de enseñar encuentra correspondencia en la adquisición de conocimiento de los estudiantes. Por su parte, en el caso de los estudiantes, resulta en un desafío quizá mayor: reconocer la existencia simultánea de dos públicos - otros estudiantes y el profesor -, ajustando su comportamiento para satisfacer las expectativas de uno sin alienar en el proceso al otro.

Si se examinan las principales herramientas empleadas ahora en la educación a distancia, es posible observar el predominio de plataforma de telecomunicación de audio y video, como Zoom, Meet, Cisco Webex y Microsoft Teams. Dichas plataformas se caracterizan por proveer una base para videoconferencias grupales, en que profesores y estudiantes pueden participar, tanto activando micrófonos y cámaras de video, compartiendo pantalla o enviando mensajes de texto, o simplemente estando presentes, dando lugar al "fenómeno de las pantallas negras" asociado en algunos casos a la inestabilidad de las conexiones y en otras a un gesto voluntario de bloquear la opción de ser visto por otros.

En cualquiera de estos casos, el uso de estas herramientas suele ir la mano de una restricción de las posibilidades de percepción. La utilidad pedagógica de los sentidos del tacto, gusto u olfato es inmediatamente restringida en el contexto de la enseñanza a distancia, siendo ahora asequible solo de manera indirecta, reflexiva y producto de la interrogación sobre las sensaciones individuales o mediante el uso de una planificación didáctica detallada que permita generarlas en común pese a la distancia. De la misma manera, la información visual es reducida, limitándose ahora al movimiento de partes específicas del cuerpo - usualmente el cuello y el rostro - y sus correlatos auditivos, si cámaras y micrófonos están disponibles, disminuyéndose por tanto la presión del reconocimiento de ser observado y, especialmente relevante desde el lado del docente, la capacidad de aprovechar eventos fortuitos como una alternativa para enseñar unos contenidos (Luhmann, 2004b). La interacción por tanto se ritualiza y, salvo el uso de metodologías explícitamente diseñadas para enfrentar estos desafíos, pierde su potencial original propio de la presencialidad.

\section{La pandemia por COVID 19: una oportunidad para la reflexión educacional}

Como se ve a partir de este examen, la pandemia por COVID 19 ha resultado en un desafío inmenso para los procesos de enseñanza y aprendizaje. Dicho reto ha sido ya reconocido en la literatura especializada, subrayándose sus impactos en términos de acceso, equidad y 
calidad, especialmente en grupos socioeconómicos que carecen de condiciones residenciales, infraestructura tecnológica, competencias y habilidades y el entorno familiar necesario para adaptarse a este nuevo entorno de enseñanza (Klerk; Palmer, 2021; Álvarez; Labraña; Brunner, 2021; Teng; Wu, 2021; Lepp et al., 2021).

En este sentido, precisamente por la urgencia del cambio, es preciso examinar los impactos de las nuevas tecnologías en los procesos de enseñanza y aprendizaje. A nivel de las organizaciones del sistema educativo, principalmente escuelas, institutos técnico profesionales y universidades, es preciso definir qué significa el uso de metodologías de enseñanza en línea y cuáles son las condiciones tanto habilitantes como restrictivas que esto abre para el éxito de los procesos de docencia y aprendizaje. En efecto, si se examinan las tecnologías hoy empleadas en el proceso de enseñanza y aprendizaje por la pandemia por COVID 19, es posible observar que ambas no son capaces de replicar la ruptura entre espacio familiar y educativo ni la complejidad de los procesos de percepción característicos de las interacciones cara-a-cara en instituciones educativas. Por lo tanto, la identificación de las posibilidades docentes de estas herramientas no debe buscarse en su capacidad de asemejarse a la presencialidad física, estándar frente al cual salvo mediante innovaciones tecnológicas aceleradas y con rápida capacidad de generalización a nivel social - siempre palidecerán en comparación con la interacción cara-a-cara, sino en el aprovechamiento de las alternativas que este medio en particular ofrece para las comunicaciones pedagógicas y que la diferencia de la copresencialidad.

Existen en este sentido una serie de aspectos positivos de la enseñanza a distancia ya identificados en la literatura especializada: su mayor accesibilidad debido a la ausencia de tiempos organizacionales predefinidos, su independencia respecto del espacio y las posibilidades que ofrece para la creación de comunidades de enseñanza globales, su capacidad de incentivar procesos de autoaprendizaje a lo largo de la vida adecuados a las necesidades de cada alumno y, no menos importante, su menor costo económico (Altmann, 2019). Cabría entonces examinar, en el interior de cada organización y diferenciando según áreas del conocimiento involucradas, en qué medida dichas ventajas pueden ser empleadas para la consecución de los fines formativos en ausencia de la posibilidad de aprovechar plenamente las ventajas pedagógicas de la interacción cara-a-cara (Tesar, 2020; Çalıkoğlu; Gümüş, 2021; Yang; Huang, 2021).

Lo anterior plantea también desafíos de acceso y equidad respecto de temáticas cuya relevancia, si bien no se han considerado en mayor profundidad en este análisis, afectan el potencial de estos medios de enseñanza. Debido a la emergencia de la transición a la educación a distancia aún hoy existen comunidades educativas que carecen de las condiciones de conectividad mínimas para el desarrollo de las clases en línea (Almaiah; Al-Khasawneh; 
Althunibat, 2020). Este problema de equidad afecta especialmente a los estudiantes de los niveles iniciales y secundarios, donde las actividades docentes en ya más de un año de pandemia se han visto reducidas en muchos casos a la entrega de "guías y material para la casa" con alternativas de retroalimentación más reducidas aún.

Ocupamos en este sentido de reflexión situada organizacionalmente en tanto no existe una respuesta adecuada para todas las circunstancias. Es preciso entonces incentivar internamente procesos de diálogo que incorporen a todos los actores involucrados mediante técnicas de discusión participativa para, de esta manera, identificar líneas de acción que permitan incorporar estas tecnologías en la docencia, aprovechando sus ventajas particulares y complementando, mediante modalidades mixtas, las restricciones que posee este medio.

\section{Conclusiones}

En el presente ensayo hemos explorado los desafíos que la pandemia por COVID 19 ha tenido sobre el sistema educativo con su acelerada presión por transitar hacia formas de enseñanza a distancia. Con este fin, empleamos elementos de la teoría de sistemas sociales para identificar los atributos del sistema educativo que explican su extraordinaria dependencia de la interacción cara-a-cara en comparación con otros sistemas parciales. Nuestro análisis sugiere que la enseñanza a distancia, al menos considerando los desarrollos tecnológicos hoy dominantes, enfrenta dos retos: la ausencia de un espacio de interacción específicamente educativo y la restricción de la enseñanza a aquello que puede ser visto u oído con la limitación de las resultantes posibilidades de autoeducación.

Numerosas líneas de investigación se articulan producto de lo anterior. En términos conceptuales la importancia de la interacción cara-a-cara en la educación ejemplifica la centralidad del espacio en las dinámicas sociales, aspecto usualmente pasado por alto desde la teoría de sistemas. En efecto, en el planteamiento original de Niklas Luhmann, la reproducción de los sistemas sociales se realiza en las dimensiones objetual, temporal y social, sin una mención explícita a la dimensión espacial (Galindo, 2007). Si bien posteriores autores han desarrollado esta idea, subrayando la relevancia de la distinción cerca/lejos en la comunicación, dichos debates han sido realizados hasta ahora principalmente en términos conceptuales sin asociarse a perspectivas empíricas de investigación (Stichweh, 1998). La reflexión avanzada permite en cambio comprender el espacio como un médium en que, si bien diferentes formas pedagógicas pueden realizarse, posiciona una serie de determinaciones, tanto restrictivas como habilitantes, resultado del médium seleccionado (véase Urquieta; Maríñez; Jorquera, 2017). Si se 
considera esto se abre una interesante línea de estudios, informada teóricamente, sobre el impacto de la digitalización de la enseñanza y el aprendizaje tanto en el plano organizacional, relacionado con la gestión de la docencia y la evaluación, como en el plano interaccional, referido a los procesos de socialización en contextos educacionales.

Otro tipo de reflexión que deriva de esta línea es como la posibilidad de disponer de espacios físicos diferenciados, aporta en la estabilización de la diferenciación funcional en tanto la separación entre el espacio productivo, en este caso educativo, del espacio del hogar permite la realización efectiva de la función educativa manteniendo separadas las tareas domésticas de lo educativo.

Por otro lado, desde una perspectiva interesada en buenas prácticas, es necesario identificar el rol que juega el uso de diferentes metodologías didácticas en el aprovechamiento de las posibilidades de enseñanza y aprendizaje en el contexto de la formación a distancia. En este punto cabe examinar de qué manera el uso de diferentes metodologías puede contribuir a atenuar o incluso superar los problemas presentados como característicos de la interacción educativa a distancia. La investigación orientada empíricamente y, en particular, aquella centrada en las experiencias de aprendizaje de los estudiantes en el contexto de la pandemia por COVID 19 es entonces fundamental, especialmente en relación con la formación de profesores con las competencias adecuadas para hacer uso de las nuevas tecnologías y la re-estructuración del currículo en un sentido que permita aprovechar las posibilidades de estos medios considerando en particular las características de las materias y las demandas de los distintos estudiantes.

En una dirección similar, desde el lado del estudiante, cabría examinar en qué medida la posibilidad de disponer de espacios físicos diferenciados puede contribuir a atenuar la ausencia de ruptura entre el espacio familiar y el educativo. Si el análisis desarrollado en este artículo es correcto, si bien la existencia de un espacio pedagógico separado puede resolver parte de los problemas aquí identificados, manteniendo separadas las tareas domésticas de las pedagógicas, no puede superar los límites del modo de interacción característico de los procesos de enseñanza y aprendizaje fundados en medios digitales.

Para finalizar es necesaria una nota de cautela. La teoría de sistemas sociales es en principio una teoría autorreferencial que no teme aplicar sus enunciados sobre la realidad a sí misma. Siguiendo esta línea, diversos autores han notado que la teoría de sistemas es en sentido estricto una teoría de la modernidad sobre sí misma (Baecker, 2006). El presente ensayo - en tanto discusión de las posibilidades de las tecnologías usadas en el proceso de enseñanza y aprendizaje por la pandemia por COVID 19 - no escapa a esta consideración. Las restricciones aquí exploradas son entonces limitaciones de las tecnologías empleadas y nada existe contra la 
idea que, a medida que ellas respondan a la complejidad de la interacción, no puedan superarlas. Para ese futuro solo cabe esperar y, desde las ciencias sociales, desarrollar las herramientas teóricas para su entendimiento.

\section{Referencias}

ADEDOYIN, Olasile Babatunde; SOYKAN, Emrah (2020), "Covid-19 pandemic and online learning: the challenges and opportunities". Interactive Learning Environments, pp. 1-13.

AGUILERA-HERMIDA, Patricia (2020), "College students use and acceptance of emergency online learning due to COVID-19”. International Journal of Educational Research Open, v. 1.

ALMAIAH, Mohammed; AL-KHASAWNEH, Ahmad; ALTHUNIBAT, Ahmad (2020), "Exploring the critical challenges and factors influencing the E-learning system usage during COVID-19 pandemic". Education and Information Technologies, pp. 1-20.

ALTMANN, Andreas; EBERSBERGER, Bernd; MÖSSENLECHNER, Claudia; WIESER, Desiree (Orgs.). (2019), The disruptive power of online education: Challenges, opportunities, responses. Austria, Emerald Publishing.

ÁLVAREZ, Javier; LABRAÑA, Julio; BRUNNER, José Joaquín (2021), "La educación superior técnico profesional frente a nuevos desafíos: La Cuarta Revolución Industrial y la Pandemia por COVID-19". Revista Educación, Política Y Sociedad, v. 6, n. 1, pp. 11-38.

ATILES, Julia; ALMODÓVAR, Mayra; CHAVARRÍA VARGAS, Aleida; DIAS, María; ZÚÑIGA LEÓN, Irma (2021), "International responses to COVID-19: challenges faced by early childhood professionals”. European Early Childhood Education Research Journal, v. 29, n. 1, pp. 66-78.

BAECKER, Dirk (2006), "Niklas Luhmann in the Society of the Computer". Cybernetics \& Human Knowing: A Journal of Second-Order Cybernetics, Autopoiesis, and Cyber-Semiotics, v. 13, pp. 2540 .

BHAGAT, Sarbottam; KIM, Dan (2020), "Higher Education Amidst COVID-19: Challenges and Silver Lining”. Information Systems Management, v. 37, n. 4, pp. 366-371.

ÇALIKOĞLU, Alper; GÜMÜŞ, Sedat (2021), “The Future of Higher Education: The Effects of Covid-19 on Teaching, Research and Internationalization”. Yuksekogretim Dergisi, v. 10, n. 3, pp. 249-259.

CARRILLO, Carmen; FLORES, María Assunção (2020), "COVID-19 and teacher education: a literature review of online teaching and learning practices". European Journal of Teacher Education, v. 43, n. 4, pp. 466-487.

COOLICAN, Mariana, BORRAS, Juan Carlos; STRONG, Michael (2020), "Argentina and the COVID19: Lessons learned from education and technical colleges in Buenos Aires Province". Journal of Education for Teaching, v. 46, n. 4, pp. 484-496.

FORMOSINHO, João (2021), "From schoolification of children to schoolification of parents? educational policies in COVID times". European Early Childhood Education Research Journal, v. 29, n. 1, pp. 141-152.

GALINDO, Jorge (2007), "La teoría sistémica de la sociedad de Niklas Luhmann: alcances y límites", in N. Luhmann (Ed.), La sociedad de la sociedad. México, Editorial Herder, pp. XXIII-XLV. 
GIROUX, Henry (2014), Neoliberalism's war on higher education. Chicago, Illinois. Haymarket Books.

HEYANG, Tuomeiciren \& MARTIN, Rose (2020), "A reimagined world: international tertiary dance education in light of COVID-19”. Research in Dance Education, pp. 1-15.

HORVITZ, Brian (2017), "Future Directions for Research on Online Technical Education”. Community College Journal of Research and Practice, v. 41, n. 6, pp. 381-385.

KLERK, E. D., \& PALMER, J. M. (2021), Resetting education priorities during COVID-19: Towards equitable learning opportunities through inclusion and equity. Perspectives in Education, v. 39, n. 1, pp. 12-28.

KOŽIĆ, Danijela, GLOBOČNIK, Ana; BAKIĆ-TOMIĆ, Ljubica (2013), "Use of non-verbal communication channels in the classroom". Croatian Journal of Education, v. 15, p. 1, pp. 141-153.

LARREAMENDY-JOERNS, Jorge \& LEINHARDT, Gaea (2006), "Going the Distance With Online Education". Review of Educational Research, v. 76, n. 4, pp. 567-605.

LEPP, Liina, AAVIKU, Triinu, LEIJEN, Äli, PEDASTE, Margus; SAKS, Katrin (2021), "Teaching during COVID-19: The Decisions Made in Teaching”. Education Sciences, v. 11, n. 2, pp. 1-21

LUHMANN, Niklas (2007), La sociedad de la sociedad. México, Editorial Herder.

LUHMANN, Niklas (1975), "Interaktion, Organisation, Gesellschaft”, in N. Luhmann (Ed.), Soziologische Aufklärung 2: Aufsätze zur Theorie der Gesellschaft. Berlin, VS Verlag für Sozialwissenschaften, pp. 9-24.

LUHMANN, Niklas (1993), "Gesellschaftliche Struktur und semantische Tradition", in N. Luhmann (Ed.), Gesellschaftsstruktur und Semantik: Studien zur Wissenssoziologie der modernen Gesellschaft. Frankfurt, Suhrkamp, pp. 9-71.

LUHMANN, Niklas (1998), "Educación y socialización", in N. Luhmann (Ed.), Complejidad y modernidad: De la unidad a la diferencia. Madrid, Editorial Trotta, pp. 177-185.

LUHMANN, Niklas (2002), Das Erziehungssystem der Gesellschaft. Frankfurt, Suhrkamp.

LUHMANN, Niklas (2004), "Erziehender Unterricht als Interaktionssystem (1985)", in N. Luhmann (Ed.), Schriften zur Pädagogik. Frankfurt, Suhrkamp Verlag, pp. 11-22.

LUHMANN, Niklas (2004), "Strukturelle Defizite: Bemerkungen zur systemtheoretischen Analyse des Erziehungssystem (1987)", in N. Luhmann (Ed.), Schriften zur Pädagogik. Frankfurt, Suhrkamp Verlag, pp. 91-110.

LUHMANN, Niklas; SCHORR, Karl (2000), Problems of reflection in the system of education. Münster, Waxmann.

PARSONS, Talcott (1959), "The school class as a social system: Some of its functions in American society". Harvard Educational Review, v. 29, n. 4, pp. 298-318.

PIETRAß, Manuela (2017), "Was ist das Neue an „digitaler Bildung“? Zum hochschuldidaktischen Potenzial der elektronischen Medien”. Erziehungswissenschaft, v. 28, n. 2, pp. 19-27.

QUINTANA AVELLO, Ingrid (2020), "Covid-19 y Cierre de Universidades ¿Preparados para una Educación a Distancia de Calidad?". Revista Internacional De Educación Para La Justicia Social, v. 9, n. 3, pp. 1-11. 
REIMERS, Fernando; SCHLEICHER, Andreas (2020), Un marco para guiar una respuesta educativa a la pandemia del 2020 del COVID-19. Perú, Enseña Perú.

SHAH, D. (2016), "Online education: Should we take it seriously?". Climacteric: The Journal of the International Menopause Society, v. 19, n. 1, pp. 3-6.

STICHWEH, Rudolph (1998), "Raum, Region und Stadt in der Systemtheorie”. Soziale Systeme, v. 4, n. 2, pp. 341-358.

TENG, Mark Feng; WU, Junjie Gavin (2021), "Tea or tears: online teaching during the COVID-19 pandemic". Journal of Education for Teaching, v. 47, n. 2, pp. 290-292.

TESAR, Marek (2020), “Towards a Post-Covid-19 'New Normality?': Physical and Social Distancing, the Move to Online and Higher Education". Policy Futures in Education, v. 18, n. 5, pp. 556-559.

TYRELL, Hartmann; VANDERSTRAETEN, Raf (2007), "Familie und Schule: zwei Orte der Erziehung", in J. Aderhold \& O. Kranz (Eds.), Intention und Funktion: Probleme der Vermittlung psychischer und sozialer Systeme. Berlin, VS Verlag für Sozialwissenschaften, pp. 159-174.

URQUIETA, María Antonieta; MARÍÑEZ, César; JORQUERA, Catalina (2017), “Territorio como medium: Discusión sobre rendimientos analíticos para las observaciones de la complejidad socioespacial”. Revista Mad, v. 37, pp. 143-167.

VANDERSTRAETEN, Raf (2002), "The autopoiesis of educational organizations: the impact of the organizational setting on educational interaction”. Systems Research and Behavioral Science, v. 19, n. 3 , pp. 243-253.

VANDERSTRAETEN, Raf (2004a), "The social differentiation of the educational system". Sociology, v. 38 , n. 2, pp. 255-272.

VANDERSTRAETEN, Raf (2004b), "Erziehung als Kommunikation. Doppelte Kontingenz als systemtheoretischer Grundbegriff", in D. Lenzen (Ed.), Suhrkamp Taschenbuch Wissenschaft: Vol. 1657. Irritationen des Erziehungssystems: Pädagogische Resonanzen auf Niklas Luhmann. Frankfurt, Suhrkamp, pp. 37-64.

VANDERSTRAETEN, Raf (2004c), "Education and Society: a Plea for a istoricised Approach". Journal of Philosophy of Education, v. 38, n. 2, pp. 195-206.

VANDERSTRAETEN, Raf (2020), “How does education function?”. European Educational Research Journal, pp. 1-11

WINTERS, Niall; PATEL, Kunal (2021), "Can a reconceptualization of online training be part of the solution to addressing the COVID-19 pandemic?". Journal of Interprofessional Care, v. 35, n. 2, pp. 161-163.

YANG, Bin; HUANG, Cheng (2021), "Turn crisis into opportunity in response to COVID-19: experience from a Chinese University and future prospects". Studies in Higher Education, vol. 46, n. 1, pp. 121132.

ZAKHAROVA, Ulyana; VILKOVA, Ksenia; EGOROV, Gennadiy (2021), "It Can't Be Taught Online: Applied Sciences during the Pandemic". Voprosy Obrazovaniya / Educational Studies Moscow, v. 1, pp. 115-137. 


\begin{abstract}
The COVID 19 pandemic has significantly altered the functioning of educational institutions. Most governments have adopted social distancing and quarantine strategies, with the suspension of face-to-face classes being one of the most frequently used measures. This essay seeks to address online education using elements of the theory of social systems developed by Niklas Luhmann. According to our analysis, these teaching modalities face at least two challenges: a) the absence of a specifically educational interaction space different from the family space and b) the restriction of teaching to what can be transmitted digitally. This challenge demands the need to promote organizational processes in educational institutions that recognize the possibilities of distance learning.
\end{abstract}

Keywords: online education; interaction; formative processes; social systems theory.

\title{
Resumo
}

A pandemia COVID 19 alterou significativamente o funcionamento das instituições educacionais. A maioria dos governos tem adotado estratégias de distanciamento social e quarentena, sendo a suspensão das aulas presenciais uma das medidas mais utilizadas. Este ensaio busca abordar a educação online a partir de elementos da teoria dos sistemas sociais desenvolvida por Niklas Luhmann. De acordo com nossa análise, essas modalidades de ensino enfrentam pelo menos dois desafios: a) a ausência de um espaço especificamente educativo de interação diferente do espaço familiar eb) a restrição do ensino ao que pode ser transmitido digitalmente. $O$ exposto exige a necessidade de promover processos organizacionais nas instituições de ensino que reconheçam as possibilidades da educação a distância.

Palavras-chave: educação online; interação; processos formativos; teoria dos sistemas sociais. 